\title{
BUCKLING OF DOUBLE-WALLED CARBON NANOTUBES
}

\author{
Isaac Elishakoff ${ }^{1}$, Kévin Dujat ${ }^{2}$, Maurice Lemaire ${ }^{2}$ \\ ${ }^{1}$ Department of Mechanical Engineering, Florida Atlantic University, USA \\ ${ }^{2}$ French Institute for Advanced Mechanics, Aubière, France
}

\begin{abstract}
In this note we deal with the approximate solution of the buckling problem of a clamped-free double-walled carbon nanotube. First the finite difference method is utilized to solve this case. Then this approach is verified by solving the buckling problem of a double-walled carbon nanotube that is simply supported at both ends for which the exact solution is available.
\end{abstract}

Keyword: Buckling, nanotube, finite difference method, clamped-free.

\section{INTRODUCTION}

The studies on buckling of carbon nanotubes (CNTs) include those of Yakobson et al. [1], Cornwell and Wille [2], Yao and Lordi [3], Garg et al. [4], Lu et al. [5], Wang et al. [6], Falvo et al. [7], Guo et al. [8], Sudak [9], He et al. [10] and Wang [11,12]. However, buckling of carbon nanotubes is not yet studied sufficiently. For example, we are not able to conclude yet if this kind of a structure exhibits the same buckling behavior as uniform beams. In their work on the buckling of double-walled carbon nanotubes (DWCNTs) Elishakoff and Pentaras [13] found, with Galerkin method, that the ratio between the critical loads of a clamped-clamped DWCNT and one simply supported at both ends is about four whereas the analogous ratio for the uniform beam equals four exactly. They did not investigate the case of clamped-free DWCNTs because of the necessity to satisfy different boundary conditions for inner and outer nanotubes. This study fills the existing gap.

Hereinafter, we use the finite difference method that has been widely used in the past for buckling analysis of various structures (see for example, works by Salvadori [14], Iremonger [15], Chajes [16], and Mikhailov [17]).

\section{BUCKLING DIFFERENTIAL EQUATIONS IN FINITE DIFFERENCE FORM}

The governing differential equations for buckling of the DWCNTs read [13]:

$$
c\left(w_{2}-w_{1}\right)=E I_{1} \frac{d^{4} w_{1}}{d x^{4}}+P \frac{A_{1}}{A_{1}+A_{2}} \frac{d^{2} w_{1}}{d x^{2}}
$$




$$
-c\left(w_{2}-w_{1}\right)=E I_{2} \frac{d^{4} w_{2}}{d x^{4}}+P \frac{A_{2}}{A_{1}+A_{2}} \frac{d^{2} w_{2}}{d x^{2}}
$$

where $x$ is the axial coordinate, $w_{i}(x, t)$ is the transverse displacement of the $i^{\text {th }}$ tube, $c$ $=71.11 \mathrm{GPa}^{3}$ is the Van der Waals interlayer interaction coefficient, $E=1 \mathrm{TPa}$ is the modulus of elasticity, inner radius $R_{1}$ is taken as $0.35 \mathrm{~nm}$, whereas the outer radius $R_{2}$ is fixed at $0.7 \mathrm{~nm}$, the thickness of each tube is $0.34 \mathrm{~nm}$. The sought buckling load is denoted as $P, I_{i}$ is the second moment of area of the $i^{\text {th }}$ tube, and $A_{i}$ is the cross-sectional area; the indexes $i=1,2$ pertain to the inner tube and outer tube, respectively.

In the finite difference method expressions for the derivatives of the displacement at a point are approximated by an algebraic formula through the displacements at that point and at some nearby points. In fact, the beam is divided in $N$ segments and thus has $N+1$ nodes for which we have to find the displacement in order to know the one of the entire DWCNT when $P$ reaches the critical value.

For the first derivative we use the central difference expression:

$$
\frac{d w}{d x}\left(x_{j}\right)=\Delta^{c} w_{j}=\frac{w^{(j+1)}-w^{(j-1)}}{2 h}
$$

where $j$ is the number of the node, of coordinate $x_{j}$, where the displacement is expressed, and $h=L / N$ represents the length of a segment.

The other derivatives are expressed from the first one; thus Eqs. (1) and (2) are now written at each node $j$ as follows

$$
\begin{aligned}
c\left(w_{2}^{(j)}-w_{1}^{(j)}\right)= & \frac{E I_{1}}{h^{4}}\left(w_{1}^{(j+2)}-4 w_{1}^{(j+1)}+6 w_{1}^{(j)}-4 w_{1}^{(j-1)}+w_{1}^{(j-2)}\right)+ \\
& \frac{P A_{1}}{A_{1}+A_{2}} \frac{1}{h^{2}}\left(w_{1}^{(j+1)}-2 w_{1}^{(j)}+w_{1}^{(j-1)}\right) \\
-c\left(w_{2}^{(j)}-w_{1}^{(j)}\right)= & \frac{E I_{2}}{h^{4}}\left(w_{2}^{(j+2)}-4 w_{2}^{(j+1)}+6 w_{2}^{(j)}-4 w_{2}^{(j-1)}+w_{2}^{(j-2)}\right)+ \\
& \frac{P A_{2}}{A_{1}+A_{2}} \frac{1}{h^{2}}\left(w_{2}^{(j+1)}-2 w_{2}^{(j)}+w_{2}^{(j-1)}\right)
\end{aligned}
$$

Eqs. (4) and (5) are rewritten as follows:

$$
\begin{gathered}
\left(w_{1}^{(j+2)}-4 w_{1}^{(j+1)}+6 w_{1}^{(j)}-4 w_{1}^{(j-1)}+w_{1}^{(j-2)}\right)+ \\
\alpha_{1}\left(w_{1}^{(j+1)}-2 w_{1}^{(j)}+w_{1}^{(j-1)}\right)-c_{1}\left(w_{2}^{(j)}-w_{1}^{(j)}\right)=0 \\
\left(w_{2}^{(j+2)}-4 w_{2}^{(j+1)}+6 w_{2}^{(j)}-4 w_{2}^{(j-1)}+w_{2}^{(j-2)}\right)+ \\
\alpha_{2}\left(w_{2}^{(j+1)}-2 w_{2}^{(j)}+w_{2}^{(j-1)}\right)-c_{2}\left(w_{2}^{(j)}-w_{1}^{(j)}\right)=0
\end{gathered}
$$

where the coefficients are defined as:

$$
\begin{aligned}
& c_{i}=\frac{c h^{4}}{E I_{i}} \\
& \alpha_{i}=\frac{P A_{i} h^{2}}{E I_{i}\left(A_{1}+A_{2}\right)}
\end{aligned}
$$


The solution is found by solving the system of equations written at each node of the beam.

\section{BUCKLING OF CLAMPED-FREE DWCNTS}

Let us deal with the buckling load of a clamped-free DWCNT. The boundary conditions are:

$$
\begin{aligned}
& w_{i}(0)=w_{i}^{\prime}(0)=0 \\
& w_{i}^{\prime \prime}(L)=0 \\
& E I_{i} w^{\prime \prime \prime}{ }_{i}(L)+\frac{P A_{i}}{A_{1}+A_{2}} w_{i}^{\prime}(L)=0
\end{aligned}
$$

Thus there are 8 boundary conditions. The expression (10) leads in finite difference form to the following expressions

$$
\begin{aligned}
& w_{i}^{(0)}=0 \\
& w_{i}^{(1)}=w_{i}^{(-1)} .
\end{aligned}
$$

From Eq. (11) we get

Finally Eq. (12) provides

$$
w_{i}^{(n+1)}=2 w_{i}^{(n)}-w_{i}^{(n-1)}
$$

$$
w_{i}^{(n+2)}=2 w_{i}^{(n+1)}-2 w_{i}^{(n-1)}+w_{i}^{(n-2)}-\alpha_{i}\left(w_{i}^{(n+1)}-w_{i}^{(n-1)}\right)
$$

One observes that Eq. (16) constitutes a condition on $w_{i}^{(n+2)}$. Thus, the axis is extended over the end nodes 0 and $N$ to introduce fictitious nodes $N+1$ and $N+2$. This is done to satisfy the equations which need the displacement values at $x=-h, x=L+h$, and $x=L+2 h$ (Fig. 1). We consider the equations of the displacement expressed from $j=1$ to $j=N$ in order to have $2 N$ equations and variables.

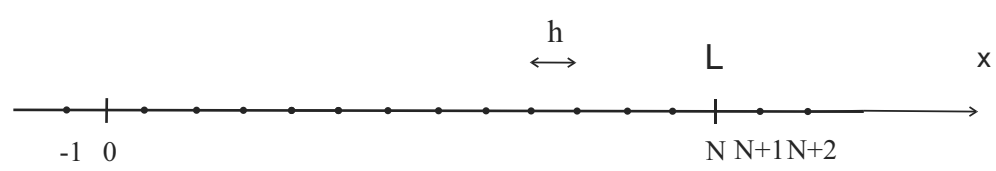

Fig. 1. Nanotube divided for finite difference analysis

In the general case when the beam is divided into $N$ segments, we have to satisfy, at each nodal point $j$, the difference equations (6) and (7), which correspond, for $i=1,2$ to the following expression:

$$
w_{i}^{(j-2)}-\left(4-\alpha_{i}\right) w_{i}^{(j-1)}+\left(6+2 \alpha_{i}+c_{i}\right) w_{i}^{(j)}-\left(4-\alpha_{i}\right) w_{i}^{(j+1)}+w_{i}^{(j+2)}-c_{i} w_{1+\delta_{i, 1}}^{(j)}=0
$$

where $\delta_{i, 1}$ is the Kronecker's delta. The latter equals unity when $i=1$ and vanishes otherwise. The coefficients in Eq. (17) are constant and only the parameter $\alpha_{i}$ is unknown. The coefficients are sorted in a $N \times N$ matrix, and since its determinant must vanish we obtain the value of the critical load as the smallest root. 
For illustration consider the cases $N=2$ and $N=3$ in detail. The inner nodal points are 0,1 and 2. Thus, to facilitate satisfaction of Eq. (16) we introduce fictitious nodes $-1,3$ and 4 . The boundary conditions are Eqs. (13) and (14), and Eqs. (15) and (16) lead to:

$$
\begin{aligned}
& w_{i}^{(3)}=2 w_{i}^{(2)}-w_{i}^{(1)} \\
& w_{i}^{(4)}=4 w_{i}^{(2)}-4 w_{i}^{(1)}-\alpha_{i}\left(w_{i}^{(2)}-w_{i}^{(1)}\right)
\end{aligned}
$$

By substitution of $j=1$ in Eq. (17) we obtain the equation of displacement at the node 1 for the two tubes

$$
w_{i}^{(-1)}-\left(4-\alpha_{i}\right) w_{i}^{(0)}+\left(6-2 \alpha_{i}+c_{i}\right) w_{i}^{(1)}-\left(4-\alpha_{i}\right) w_{i}^{(2)}+w_{i}^{(3)}-c_{i} w_{1+\delta_{i, 1}}^{(1)}=0
$$

Applying the boundary conditions (13), (14), (18) and (19), we obtain:

$$
\left(6-2 \alpha_{i}+c_{i}\right) w_{i}^{(1)}-\left(2-\alpha_{i}\right) w_{i}^{(2)}-c_{i} w_{1+\delta_{i, 1}}^{(1)}=0
$$

At the next node $(j=2)$ we have:

$$
\left(-4+2 \alpha_{i}\right) w_{i}^{(1)}+\left(2-2 \alpha_{i}+c_{i}\right) w_{i}^{(2)}-c_{i} w_{1+\delta_{i, 1}}^{(2)}=0
$$

The coefficients of these expressions generate a matrix equation:

$$
\left[\begin{array}{cccc}
6-2 \alpha_{1}+c_{1} & -2+\alpha_{1} & -c_{1} & 0 \\
-4+2 \alpha_{1} & 2-2 \alpha_{1}+c_{1} & 0 & c_{1} \\
-c_{2} & 0 & 6-2 \alpha_{2}+c_{2} & -2+\alpha_{2} \\
0 & -c_{2} & -4+2 \alpha_{2} & 2-2 \alpha_{2}+c_{2}
\end{array}\right]\left(\begin{array}{c}
w_{1}^{(1)} \\
w_{1}^{(2)} \\
w_{2}^{(1)} \\
w_{2}^{(2)}
\end{array}\right)=\left(\begin{array}{l}
0 \\
0 \\
0 \\
0
\end{array}\right)
$$

In order this system to have a nontrivial solution, the determinant of this matrix must vanish. This requirement leads to the following equation:

$$
2.261 * 10^{33} p^{4}-7.419 * 10^{23}+2.939 * p^{2}-1.057 * 10^{16} p+4.79 * 10^{7}=0
$$

The smallest root of this expression yields the critical load: $P_{c} r=5.314 \mathrm{nN}$.

The case $N=3$ leads to 6 equations for the nodes $j=1,2,3$. These are

$$
\begin{aligned}
& \left(7-2 \alpha_{i}+c_{i}\right) w_{i}^{(1)}-\left(4-\alpha_{i}\right) w_{i}^{(2)}+w_{i}^{(3)}-c_{i} w_{1+\delta_{i, 1}}^{(1)}=0 \\
& \left(-4+\alpha_{i}\right) w_{i}^{(1)}+\left(5-2 \alpha_{i}+c_{i}\right) w_{i}^{(2)}-\left(2-\alpha_{i}\right) w_{i}^{(3)}-c_{i} w_{1+\delta_{i, 1}}^{(2)}=0 \\
& 2 w_{i}^{(1)}-\left(4-2 \alpha_{i}\right) w_{i}^{(2)}+\left(2-2 \alpha_{i}+c_{i}\right) w_{i}^{(3)}-c_{i} w_{1+\delta_{i, 1}}^{(3)}=0
\end{aligned}
$$

Eqs. (25)-(27) are equivalent to a matrix equation. Its determinant

$$
\left|\begin{array}{cccccc}
7-2 \alpha_{1}+c_{1} & -4+\alpha_{1} & 1 & -c_{1} & 0 & 0 \\
-4+\alpha_{1} & -5-2 \alpha_{1}+c_{1} & -2+\alpha_{1} & 0 & -c_{1} & 0 \\
2 & -4+2 \alpha_{1} & 2-2 \alpha_{1}+c_{1} & 0 & 0 & -c_{1} \\
-c_{2} & 0 & 0 & 7-2 \alpha_{2}+c_{2} & -4+\alpha_{2} & 1 \\
0 & -c_{2} & 0 & -4+\alpha_{2} & 5-2 \alpha_{2}+c_{2} & -2+\alpha_{2} \\
0 & 0 & -c_{2} & 2 & -4+2 \alpha_{2} & 2-2 \alpha_{2}+c_{2}
\end{array}\right|
$$


must vanish. This requirement yields the following sixth order polynomial equation for $\mathrm{P}$ : $4.422 * 10^{47} p^{6}-1.396 * 10^{43} p^{5}+6.67 * 10^{37} p^{4}-8.499 * 10^{31} p^{3}+9.632 * 10^{24} p^{2}-2.877 * 10^{17} p+1.299 * 10^{9}=0$

The value of the critical buckling load is $P_{c r}=5.469 \mathrm{nN}$.

$P_{\text {cr }}$

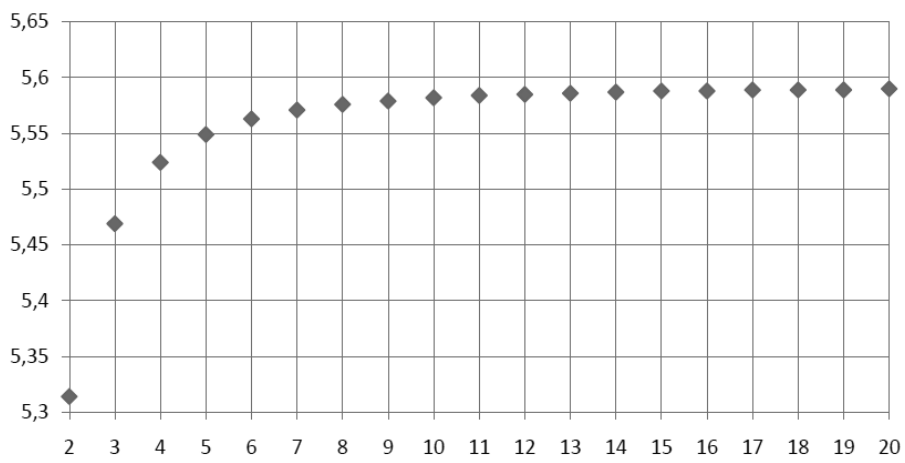

Fig. 2. Variation of the critical buckling load with the number of segments for a clamped-free DWCNT

Table 1. Critical buckling load of a clamped-free DWCNT

\begin{tabular}{|c|c|c|c|c|c|c|c|c|c|c|c|}
\hline $\mathrm{N}$ & 2 & 3 & 4 & 5 & 6 & 7 & 8 & 9 & 10 & 15 & 20 \\
\hline$P_{c r}(\mathrm{nN})$ & 5.314 & 5.469 & 5.524 & 5.549 & 5.563 & 5.571 & 5.576 & 5.579 & 5.582 & 5.588 & 5.590 \\
\hline
\end{tabular}

We also conduct the evaluation of the critical load $P$ for consecutive values of $N$ until the convergence is reached (see Fig. 2). The results are reported in Tab. 1.

\section{VERIFICATION: BUCKLING OF DWCNTS SIMPLY SUPPORTED AT BOTH ENDS}

To verify the above approach we conduct a comparison with the results previously obtained by Elishakoff and Pentaras [13] for the simply supported case. Specifically, we calculate the buckling load of a DWCNT simply supported at both ends by the finite difference method. Firstly, we write the boundary conditions of this system. For each end the following expressions should be satisfied:

$$
\begin{aligned}
& w_{i}(0)=w_{i}(L)=0, \\
& \frac{d^{2} w_{i}}{d x^{2}}(0)=\frac{d^{2} w_{i}}{d x^{2}}(L)=0 .
\end{aligned}
$$

In finite difference setting we have:

$$
w_{i}^{(0)}=w_{i}^{(N)}=0
$$


Eq.(31) leads to

$$
\frac{w_{i}^{(-1)}-2 w_{i}^{(0)}+w_{i}^{(1)}}{h^{2}}=\frac{w_{i}^{(N-1)}-2 w_{i}^{(N)}+w_{i}^{(N+1)}}{h^{2}}=0
$$

Thus we obtain two relations:

$$
\begin{aligned}
& w_{i}^{(1)}=-w_{i}^{(-1)} \\
& w_{i}^{(N+1)}=-w_{i}^{(N-1)}
\end{aligned}
$$

This time the equation of the displacement at $x=L+2 h$ is not needed so Eq. (17) is applied to nodes from $j=1$ to $j=N-1$ in order to have as many equations as the number of variables; in this case the system is of size $2(N-1)$.

$$
\left(4-2 \alpha_{i}+c_{i}\right) w_{i}^{(1)}-c_{i} w_{1+\delta_{i, 1}}^{(1)}=0
$$

This leads to the following determinant:

$$
\left|\begin{array}{cc}
4-2 \alpha_{1}+c_{1} & -c_{1} \\
-c_{2} & 4-2 \alpha_{2}+c_{2}
\end{array}\right|=0
$$

From Eq. (37) we obtain the value of the critical load as the smallest root : $P_{c r}=18.138$ $\mathrm{nN}$, whereas the Elishakoff and Pentaras [13] result is $P_{c r}=22.63 \mathrm{nN}$. One observes that 2 segments are not sufficient for an accurate evaluation of the buckling load.

In the case of three segments, i.e. $N=3$, the system is composed of 4 equations which are those at nodes $j=1$ and $j=2$ :

$$
\begin{aligned}
& \left(5-2 \alpha_{i}+c_{i}\right) w_{i}^{(1)}-\left(4-\alpha_{i}\right) w_{i}^{(2)}-c_{i} w_{1+\delta_{i, 1}}^{(1)}=0 \\
& \left(-4+\alpha_{i}\right) w_{i}^{(1)}+\left(5-2 \alpha_{i}+c_{i}\right) w_{i}^{(2)}-c_{i} w_{1+\delta_{i, 1}}^{(2)}=0
\end{aligned}
$$

Eqs. (38) and (39) lead to the following requirement:

$$
\left|\begin{array}{cccc}
5-2 \alpha_{1}+c_{1} & -4+\alpha_{1} & -c_{1} & 0 \\
-4+\alpha_{1} & 5-2 \alpha_{1}+c_{1} & 0 & -c_{1} \\
c_{2} & 0 & 5-2 \alpha_{2}+c_{2} & -4+\alpha_{2} \\
0 & -c_{2} & -4+\alpha_{2} & -5-2 \alpha_{2}+c_{2}
\end{array}\right|=0
$$

Table 2. Critical buckling load for a DWCNT simply supported at both ends

\begin{tabular}{|c|c|c|c|c|c|c|c|c|c|c|c|c|}
\hline $\mathrm{N}$ & 2 & 3 & 4 & 5 & 6 & 7 & 8 & 9 & 10 & 12 & 15 & 20 \\
\hline$P_{c r}(\mathrm{nN})$ & 18.138 & 20.403 & 21.247 & 21.646 & 21.866 & 21.999 & 22.086 & 22.146 & 22.189 & 22.245 & 22.290 & 22.326 \\
\hline
\end{tabular}

We obtain $P_{c r}=20.403 \mathrm{nN}$. The results for various values of $N$ are listed in Tab. 2. The smallest percentage-wise difference from the Elishakoff and Pentaras [13] value is 


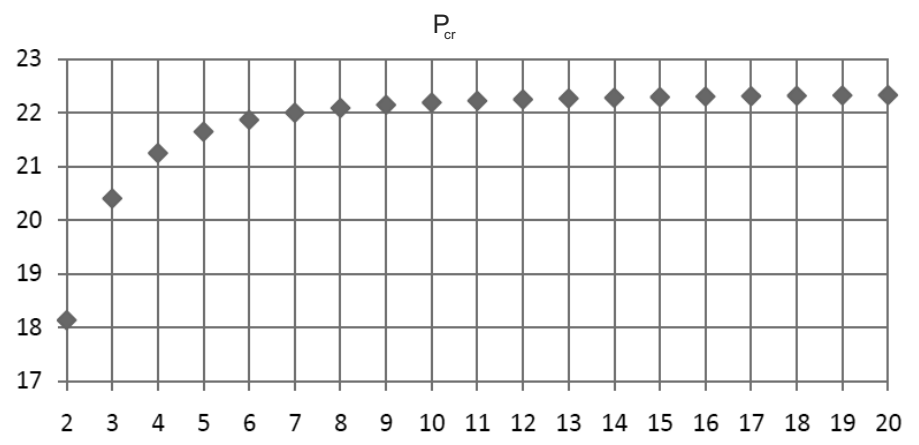

Fig. 3. Variation of the critical buckling load with the number of segments for a DWCNT simply supported at both ends

$1.34 \%$, and is achieved for $N=20$ (see Fig. 3). Thus the finite difference method yields a result that is extremely close to the exact solution.

\section{DISCUSSION AND CONCLUSION}

In this study it has been demonstrated that the finite difference method can be successfully applied to buckling of double-walled carbon nanotubes since it yields extremely reliable results. The best approximation for the clamped-free DWCNT is 5.590 (Tab. 1 ), and the best approximation for the simply supported at both ends is 22.236 (Tab. 2 ). This leads to a ratio of 3.978 , what tends to the ratio we expected from the beam theory. The difference comes from two reasons. First the convergence of the finite difference discretization; and second, the introduction of the boundary conditions. In the first case they are expressed with $w=0, w^{\prime}=0$ at the clamped edge and $w^{\prime \prime}=0$ and $w^{\prime \prime \prime}=0$ at the free edge (Eqs. 10-16). In the second case they are expressed with $w=0$ and $w^{\prime \prime}=0$ at both edges. The examination of the finite difference formulas shows that the error level would not be the same by using the third order differentiation.

The major complexity with this method is that when the number of nodes increases substantially, the calculation time increases dramatically. Indeed this process entails the solution of a large number of simultaneous equations. As indicated by Salvadori [14], one can use the Richardson's extrapolation scheme to increase the accuracy of the result instead of increasing the number of segments. This approximation is expressed by:

$$
P_{c r}=\frac{N_{1}^{2} P_{c r_{1}}-N_{2}^{2} P_{c r_{2}}}{N_{1}^{2}-N_{2}^{2}}
$$

where $N_{i}$ is the number of segments into which the beam was divided to obtain an approximation of the critical buckling load denoted as $P_{c r i}$. To illustrate the effectiveness of Richardson's extrapolation scheme, the approximate resuls obtained for the clamped-free case with $N_{1}=4$ and $N_{2}=5$ will be substituted in Eq. (41):

$$
P_{c r}=\frac{16 * 5.524-25 * 5.549}{16-25}=5.593 n N
$$


Note that this solution is nearly as accurate as can be achieved by using $N=20$ segments of the nanotubes.

Thus, the combination of the finite difference method with the Richardson's extrapolation scheme gives an excellent tool to investigate the buckling of double-walled carbon nanotubes.

\section{REFERENCES}

[1] Yakobson B. I., Brabec C. J., Berhole J., Nanomechanics of Carbon Tubes: Instabilities beyond Linear Range, Phys. Rev. Lett., 76, (1996), p. 2511.

[2] Cornwell C. F., Wille L. T., Elastic Properties of Single-Walled Carbon Nanotubes in Compression, Solid State Comm., 101 (9), (1997), p. 555.

[3] Yao N., Lordi V., Carbon Nanotube Caps as Spring: Molecular Dynamics Simulations, Phys. Rev. B., 58 (19), (1998), p. 12649.

[4] Garg A., Han J., Sinnott S. B., Interaction of Carbon-Nanotubule Proximal Probe Tips with Diamond and Graphene, Phys. Rev. Lett., 81 (11), (1998), p. 2260.

[5] Lu J.-M., Hwang C.-C., Kuo Q.-Y., Wang Y.-C., Mechanical Buckling of Multi-Walled Carbon Nanotubes: The Effects of Slenderness Ratio, Physica E, 40, (2008), p. 1305.

[6] Wang Y., Wang X.-X., Ni X.-G., Wu H.-A., Simulation of the Elastic Response and the Buckling Modes of Single-Walled Carbon Nanotubes, Comput. Mat. Sci., 32, (2005), p. 141.

[7] Falvo M. R., Clary G. J., Taylor R. M. II, Chi V., Brooks F. P. Jr, Washburn S., Superfine R., Bending and Buckling of Carbon Nanotubes under Large Strain, Nature, 389, (1997), p. 582.

[8] Guo X., Leung A. Y. T., He X. Q., Jiang H., Huang Y., Bending Buckling of Single-Walled Carbon Nanotubes by Atomic-Scale Finite Element, Composite: Part B, 39, (2008), p. 202.

[9] Sudak L. J., "Column Buckling of Multi-Walled Carbon Nanotubes Using Nonlocal Continuum Mechanism", J. Appl. Phys., 94 (11), (2003), p. 7281.

[10] He X. Q., Kitipornchai S., Liew K. M., Buckling Analysis of Multi-Walled Carbon Nanotubes: A Continuum Model Accounting for van der Waals Interaction, J. Mec. Phys. Solids, 53, (2005), p. 303.

[11] Wang Q., Hu T., Chen G., Q. Jiang, Bending Instability Characteristics of Double-Walled Carbon Nanotubes, Phys. Rev. B, 71, (2005), p. 1.

[12] Wang Q., Xu F., Zhou Q., Continuum Model for Stability Analysis of Carbon Nanotubes under Initial Bend, Int. J. Struct. Stab. Dyn., 5 (4), (2005), p. 579.

[13] Elishakoff I., Pentaras D., Buckling of a Double Walled Carbon Nanotube, Adv. Sc. Let., 2, (2009), p. 372.

[14] Salvadori M. G., Numerical Computation of Buckling Loads by Finite Difference, ASCE, 116, (1951), p. 590.

[15] Iremonger M.J., Finite difference buckling analysis of non-uniform columns, Cornp. Struct., 12, (1980), p. 741.

[16] Chajes A., Principles of Structural Stability Theory, Prentice-Hall, New York, (1974), p. 106.

[17] Mikhailov M. D., Finite Difference Method by Using Mathematica, Int. J. Heat and Mass Transfer, 37, (1994), p. 375. 